\title{
Simulated Analysis of Tooth Profile Error of Cycloid Steel Ball Planetary Transmission
}

\author{
Ruixue Hu ${ }^{1, a}$, Yuquan Zhang ${ }^{1, b, *}$, Zhanliang Zhao ${ }^{2, c}$, Liwen Zhang ${ }^{1, d}$ \\ ${ }^{1}$ Department of Automobile Engineering, Hebei Jiaotong Vocational and Technical College, No.219, \\ Zhujiang Street, Shijiazhuang, China \\ 2. Norendar International LTD. ,No.55, Yuhua East Road,Shijiazhuang, China

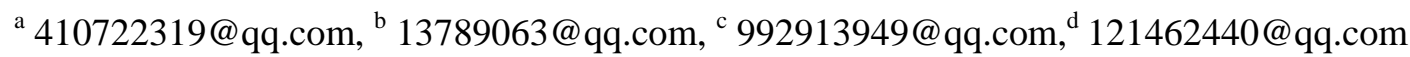 \\ *corresponding author
}

Keywords: Cycloid, planetary transmission, tooth profile error, parameter, limousine

\begin{abstract}
Cycloid steel ball planetary transmission mechanism has good prospect when being used as frequently reciprocating transmission mechanisms of precision machinery such as the windshield wiper driving mechanism of limousine. But manufacture/Installation error and tooth profile error greatly affect the error of cycloid steel ball transmission. The quantitative study of transmission accuracy affected by manufacture/ processing error can control the very error in purpose and with special emphasis, and assure the transmission to reach expected accuracy. It is also very important to study the tooth profile accuracy of cycloid plate, which is the key transmission part of cycloid steel planetary transmission, and the factors affecting the accuracy as well. This paper analyzes the relationship between the transmission mechanism's structural parameters and cycloid tooth profile error.
\end{abstract}

\section{Introduction}

With the development of modern industry and the use of modern industrial products from aerospace industry and robotic industry, etc., the mechanical transmission mechanism has developed rapidly toward the high performance direction such as high precision, high efficiency and high speed; moreover, the precision machinery require higher in both servo transmission mechanism's accuracy and other transmission performances.

Cycloid steel ball planetary transmission mechanism is a new type of mechanical transmission mechanism developed in recent years, and has good application features such as no-backlash, good carrying capacity, high meshing efficiency, large transmission ratio and wide range, compact structure, and low noise, etc. It has good prospect when being used as frequently reciprocating transmission mechanisms of precision machinery such as robots, manipulators, machine indexing mechanisms and sedan limousine. These precision transmission machinery requires higher in transmission accuracy, while the meshing error caused by tooth profile error of cycloid steel ball planetary transmission will affect the transmission accuracy obviously, therefore, the study of tooth profile error is important for the development of this kind of transmission. This paper mainly focuses on the study and analysis of the relationship between the transmission mechanism's structural parameters and cycloid tooth profile error, thus providing theoretical basis for the analysis of the manufacture and transmission accuracy of the cycloid steel ball planetary transmission.

\section{Structure and transmission principle}

The structure of the cycloid steel ball planetary transmission mechanism is as shown in Figure 1. Center plate (3) is fixed to frame (9), and its left end face is machined with outer cycloid enclosed 
groove whose tooth number (wave number) is $\mathrm{Z}_{1}$. The planetary plate's right end face is machined with inner cycloid enclosed groove whose tooth number (wave number) is $Z_{2}$. Steel ball (5) is placed inside the staggered area of the above two grooves. The right end face of plate (8) (i.e. output shaft) and left end face of plate (6) are provided with ring grooves whose numbers are the same. Steel balls (7) are placed inside the staggered area of the ring grooves; and the number of balls should be the same as that of grooves.

The drive principle is as follow: When the driving eccentric shaft (1) performs constant-speed rotation and drives plate (6) for planetary motion, the disk (6)pushes steel ball (5) to move. The ball (5) is restrained by the enclosed groove on plate(3) and pushes back the plate (6) for slow spin motion with an angle speed output by output shaft (8) through the steel ball constant-speed output mechanism (W mechanism) consisting of ring grooves on plates (6) and (8), and steel balls (7). In another word, for this transmission, the change of its moving speed is realized through the constant-speed output mechanism and meshing motion between planetary plate, center plate and steel balls.

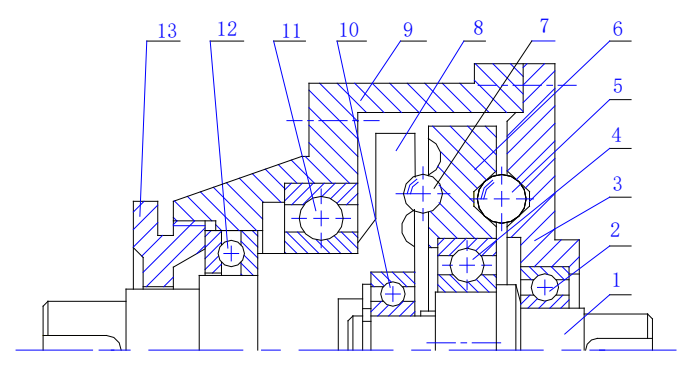

Figure 1 Structure of cycloid steel ball planetary transmission

\section{Tooth profile equation and analysis of causes of profile error}

Since the formation principle of outer cycloid and factors affecting it are the same as those of inner cycloid, this paper takes the outer cycloid as instance to study and analyze the tooth profile error affected by the error of structural parameters.

\subsection{Tooth profile equation of cycloid steel ball transmission}

The tooth profile equation will be taken as basis for study of the tooth profile error of cycloid steel ball transmission. The theoretical tooth profile equation of outer cycloid groove is shown as in:

$$
\left.\begin{array}{l}
x_{1}=\left(Z_{1}+1\right) \frac{e}{K} \cos \theta_{1}-e \cos \left[\left(1+Z_{1}\right) \theta_{1}\right] \\
y_{1}=\left(Z_{1}+1\right) \frac{e}{K} \sin \theta_{1}-e \sin \left[\left(1+Z_{1}\right) \theta_{1}\right]
\end{array}\right\}
$$

Where, e- is eccentricity;

$\theta 1$ - is rotation parameter of curve.

Figure 2 (a) is the sectional view at rotation axis, in which angle $\beta$ is the grooved angle of cycloid enclosed groove (i.e. the cone angle of cutting tool); the A and A' point paths are respectively the actual tooth profiles of plate (3) inner and outer sides; B and B' point paths are respectively the actual tooth profiles of plate (6) inner and outer sides, and $C$ and $C^{\prime}$ point paths are the theoretical tooth profiles of plate (3) and plate (6). Figure 2 (b) is an end sectional view at A'A and where it is perpendicular to rotation shaft.

Since the actual tooth profile of the cycloid groove is the equidistant line of its theoretical tooth profile, the actual tooth profile equations of the outer (inner) side of the outer cycloid groove can be deduced respectively as follows: 


$$
\left.\begin{array}{l}
x_{1}^{\prime}=\left(Z_{1}+1\right) \frac{e}{K} \cos \theta_{1}-e \cos \left[\left(1+Z_{1}\right) \theta_{1}\right] \pm r \cos \beta \cos \varphi_{1} \\
y_{1}^{\prime}=\left(Z_{1}+1\right) \frac{e}{K} \sin \theta_{1}-e \sin \left[\left(1+Z_{1}\right) \theta_{1}\right] \pm r \cos \beta \sin \varphi_{1}
\end{array}\right\}
$$

Where, "+", "-" -used respectively for outer and inner tooth profiles of outer cycloid groove; $\mathrm{r}$-steel ball radius.

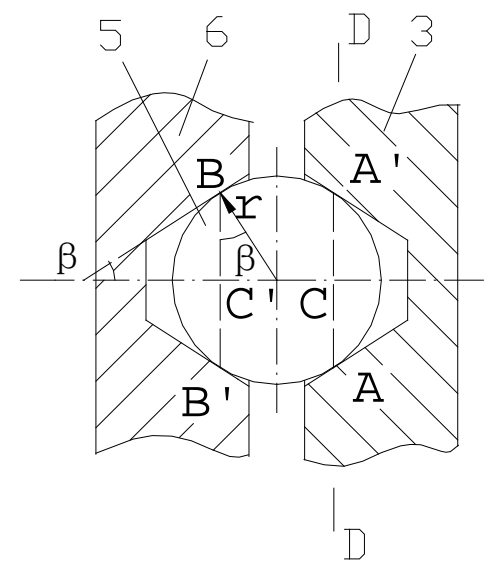

(a)

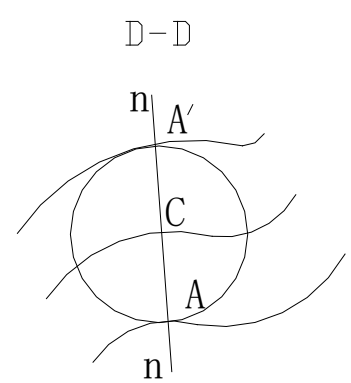

(b)

Figure 2 Sectional view of meshing countershaft

\subsection{Analysis of error causes}

From the above equation (2), we can see that the tooth profile error is mainly caused by: error of the eccentricity e, of the cycloid's curate coefficient $\mathrm{K}$, error of steel ball radius $\mathrm{r}$ (in the cycloid groove), and error of grooved angle $\beta$,other factors like the vibration during processing may cause tooth profile error as well. The actual tooth profile error is formed by the combination of various parameter errors, which is a highly nonlinear problem. As basic error analysis, this paper focuses on the tooth profile error only caused by structural parameters.

\section{Analysis of tooth profile error affected by structural parameter error}

The tooth profile error denotes the distance deviation between the actual tooth profile and the design tooth profile due to the structural parameter error in processing. Its value is defined as the deviation at the tooth profile's normal direction. According to the definition, the mathematical model of the tooth profile's normal error $\Delta L$ is established as below:

$$
\Delta L=\Delta x \cos \alpha+\Delta y \sin \alpha
$$

Where, $\Delta x, \Delta y$-- are respectively the actual tooth profile errors at $\mathrm{x}, \mathrm{y}$ coordinate directions; $\alpha$-- angle between tooth profile's normal and axis $\mathrm{x}$.

From the formula (2), tooth profile normal slope $\tan \alpha$ can be obtained, and the relationship between $\tan \alpha$ and $\sin \alpha, \cos \alpha$ can be deduced respectively:

$$
\begin{aligned}
& \sin \alpha=\frac{\mid \sin \theta_{1} / K-\sin \left[\left(1+Z_{1}\right) \theta_{1}\right]}{\sqrt{1+1 / K^{2}-2 \cos \left[\left(2+Z_{1}\right) \theta_{1}\right] / K}} \\
& \cos \alpha=\frac{\cos \theta_{1} / K-\cos \left[\left(1+Z_{1}\right) \theta_{1}\right]}{\sqrt{1+1 / K^{2}-2 \cos \left[\left(2+Z_{1}\right) \theta_{1}\right] / K}}
\end{aligned}
$$

The influence of structural parameter on tooth profile error is analyzed as below. 


\subsection{Tooth profile error $\Delta L_{e}$ caused by eccentricity error $\Delta e$}

Based on eccentricity e in the equation (2), the tooth profile errors at $\mathrm{x}, \mathrm{y}$ coordinate directions are obtained by partial differential method:

$$
\begin{aligned}
& \Delta x_{e}=\frac{\partial x_{1}^{\prime}}{\partial e} \Delta e=\left\{\left(Z_{1}+1\right) \cos \theta_{1} / K_{1}-\cos \left[\left(Z_{1}+1\right) \theta_{1}\right]\right\} \Delta e \\
& \Delta y_{e}=\frac{\partial y_{1}^{\prime}}{\partial e} \Delta e=\left\{\left(Z_{1}+1\right) \sin \theta_{1} / K_{1}-\sin \left[\left(Z_{1}+1\right) \theta_{1}\right]\right\} \Delta e
\end{aligned}
$$

According to $\Delta x_{e}$ and $\Delta y_{e}$, the tooth profile normal error $\Delta L_{e}$ can be obtained when the eccentricity e is the single error:

$\Delta L_{e}=\Delta x_{e} \cos \alpha+\Delta y_{e} \sin \alpha$

$$
=\frac{\frac{\left(1+Z_{1}\right)}{K^{2}} \cos ^{2} \theta_{1}-\frac{\left(2+Z_{1}\right) \cos \theta_{1} \cos \left[\left(1+Z_{1}\right) \theta_{1}\right]}{K}+\cos ^{2}\left[\left(1+Z_{1}\right) \theta_{1}\right]+\left\{\frac{\left(1+Z_{1}\right) \sin \theta_{1}}{K}-\sin \left[\left(1+Z_{1}\right)\right]\right\}\left|\frac{\sin \theta_{1}}{K}-\sin \left[\left(1+Z_{1}\right) \theta_{1}\right]\right|}{\sqrt{1+1 / K^{2}-2 \cos \left[\left(2+Z_{1}\right) \theta_{1}\right] / K}} \Delta e
$$

Take the parameters of the sample cycloid steel ball planetary transmission as instance, and make $\mathrm{Z}_{1}=12, \mathrm{Z}_{2}=14, \mathrm{r}=4 \mathrm{~mm}, \mathrm{k}=0.55, \mathrm{e}=1.5 \mathrm{~mm}$, and $\beta=45^{\circ}$. Take one tooth profile of the outer cycloid plate for study; when different positive values are taken respectively as $\Delta e$, a group of tooth profile normal errors $\Delta L_{e}$ can be obtained through calculation, whose curve is shown as in Figure 3. Take the curve obtained when $\Delta e=0.01 \mathrm{~mm}$ as example and analysis the influence of $\Delta e$ on tooth profile error. According to the Fig., the tooth profile normal error is smaller at the dedendum ( $b$ and $c$ in Fig.), while the normal error is larger at the addendum (a in Fig.), i.e. the amplitude of actual tooth profile becomes larger. Conversely, negative values $\Delta e$ will affect in the opposite way.

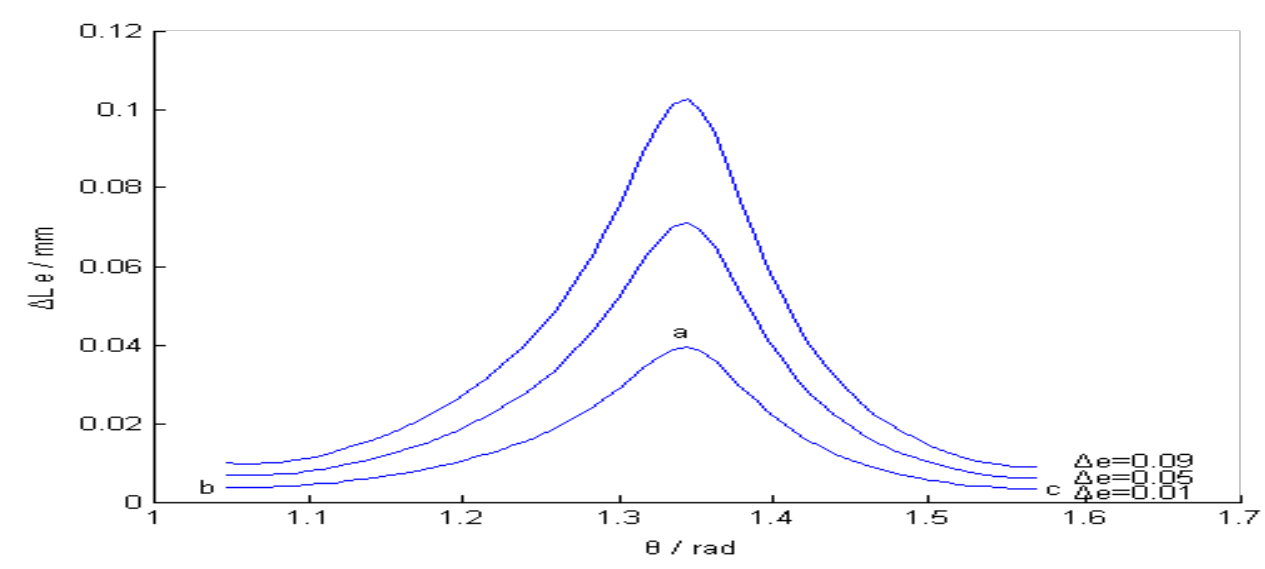

Figure $3 \Delta L_{e}$ change curve

\subsection{The error of tooth profile $\Delta L_{K}$ caused by curate coefficient error $\Delta k$}

Similarly, basing on curtate coefficient $\mathrm{K}$ in the equation (2) to obtain the actual tooth profile errors $\Delta x_{K}$ and $\Delta y_{K}$ at $\mathrm{x}, \mathrm{y}$ coordinate directions by partial differential method. Then the tooth profile normal error can be deduced when $\mathrm{K}$ is the only error.

$$
\Delta L_{K}=\frac{\left(Z_{1}+1\right) \frac{e}{K^{2}}\left\{\cos \theta_{1} \cos \left[\left(1+Z_{1}\right) \theta_{1}\right]-\frac{\cos ^{2} \theta_{1}}{K}-\sin \theta_{1} \mid \frac{\sin \theta_{1}}{K}-\sin \left[\left(Z_{1}+1\right) \theta_{1}\right]\right\}}{\sqrt{1+1 / K^{2}-2 \cos \left[\left(2+Z_{1}\right) \theta_{1}\right] / K}} \Delta K
$$

When different positive values are taken respectively as $\Delta k$, a group of tooth profile normal 
errors $\Delta L_{K}$ can be obtained through calculation, whose curve is shown as in Figure 4. Take the curve obtained when $\Delta k=0.01$ as example and analysis the influence of $\Delta k$ on tooth profile error. According to the Fig., the tooth profile normal error is smaller at the dedendum (b and c in Fig.), while the normal error is larger at the addendum (a in Fig.). Since the very error is negative value, the amplitude of actual cycloid tooth profile becomes smaller and the profile becomes gentle. Conversely, negative values $\Delta k$ will affect in the opposite way.

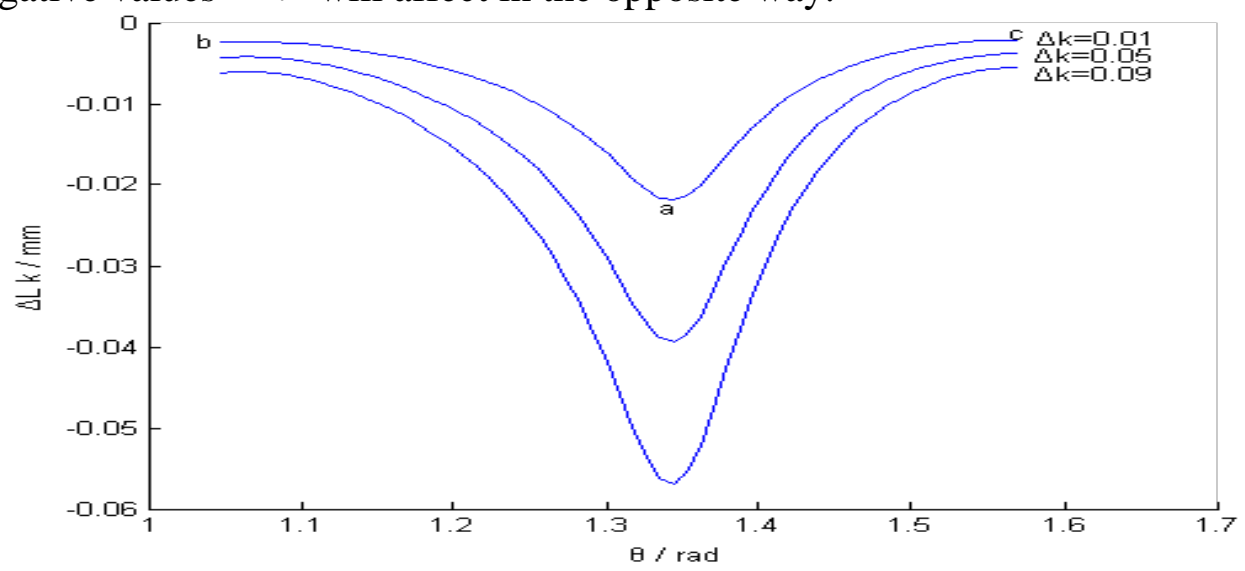

Figure $4 \Delta L_{K}$ change curve

\subsection{Tooth profile error $\Delta L_{r}$ caused by steel ball radius error $\Delta r$}

Basing on the steel ball radius $r$ in the equation (2) to obtain the actual tooth profile errors $\Delta x_{r}$ and $\Delta y_{r}$ at $\mathrm{x}, \mathrm{y}$ coordinate directions by partial differential method. Then the tooth profile normal error can be deduced when $r$ is the only error.

$$
\Delta L_{r}=\frac{\cos \varphi_{1}\left\{\cos \theta_{1} / K-\cos \left[\left(1+Z_{1}\right) \theta_{1}\right]\right\}+\sin \varphi_{1}\left|\sin \theta_{1} / K-\sin \left[\left(1+Z_{1}\right) \theta_{1}\right]\right|}{\sqrt{1+1 / K^{2}-2 \cos \left[\left(2+Z_{1}\right) \theta_{1}\right] / K}} \cos \beta \Delta r
$$

When different positive values are taken respectively as $\Delta r$, a group of tooth profile normal errors $\Delta L_{r}$ can be obtained through calculation, whose curve is shown as in Figure 5. Take the curve obtained when $\Delta r=0.01 \mathrm{~mm}$ as example and analysis the influence of $\Delta r$ on tooth profile error. According to the Fig., the tooth profile normal error is smaller at the dedendum (b and c in Fig.), while the normal error is larger at the addendum (a in Fig.), i.e. the amplitude of actual cycloid tooth profile becomes larger and the profile becomes bent. Conversely, negative values $\Delta r$ will affect in the opposite way.

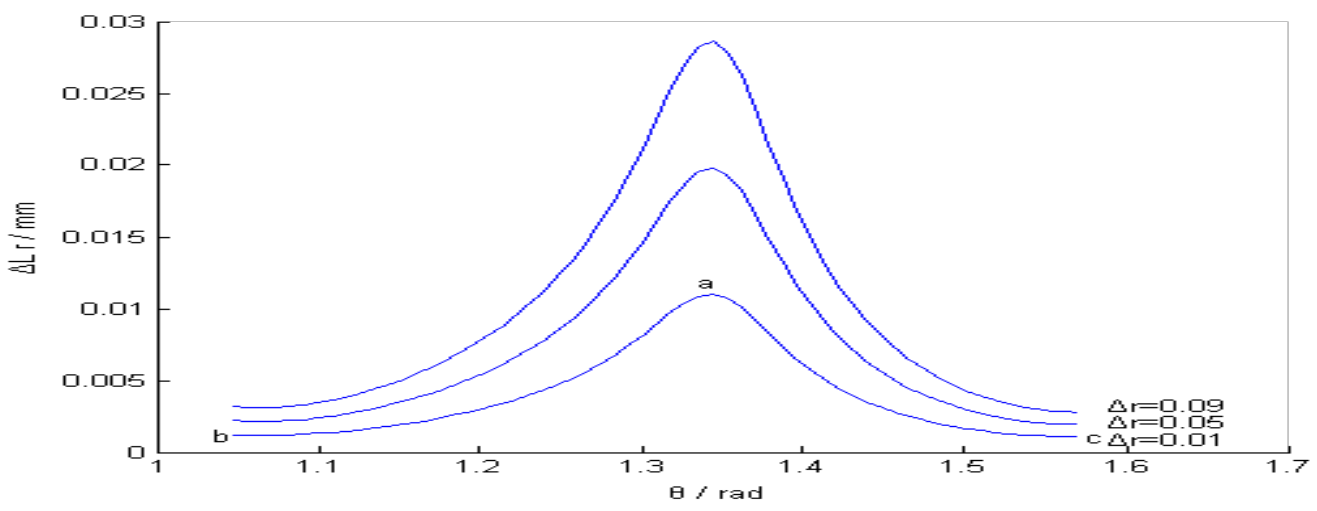

Figure $5 \Delta L_{r}$ change curve 


\subsection{Tooth profile error $\Delta L_{\beta}$ caused by grooved angle $\Delta \beta$}

Basing on the grooved angle $\beta$ in the equation (2) to obtain the actual tooth profile errors $\Delta x_{r}$ and $\Delta y_{r}$ at $\mathrm{x}, \mathrm{y}$ coordinate directions by partial differential method. Then the tooth profile normal error can be deduced when $\beta$ is the only error.

$$
\Delta L_{\beta}=\frac{\cos \varphi_{1}\left\{\cos \left[\left(1+Z_{1}\right) \theta_{1}\right]-\cos \theta_{1} / K\right\}-\sin \varphi_{1} \mid \sin \theta_{1} / K-\sin \left[\left(1+Z_{1}\right) \theta_{1}\right]}{\sqrt{1+1 / K^{2}-2 \cos \left[\left(2+Z_{1}\right) \theta_{1}\right] / K}} r \sin \beta \Delta \beta
$$

When different values are taken respectively as $\Delta \beta$, a group of tooth profile normal errors $\Delta L_{\beta}$ can be obtained through calculation, whose curve is shown as in Fig. 6. Take the curve obtained when $\Delta \beta=0.01$ radas example and analysis the influence of $\Delta \beta$ on tooth profile error. According to the Fig., the tooth profile normal error is smaller at the dedendum (b and c in Fig.), while the normal error is larger at the addendum (a in Fig.) and is of negative value, i.e. the amplitude of actual cycloid tooth profile becomes smaller. Conversely, negative values $\Delta \beta$ will affect in the opposite way.

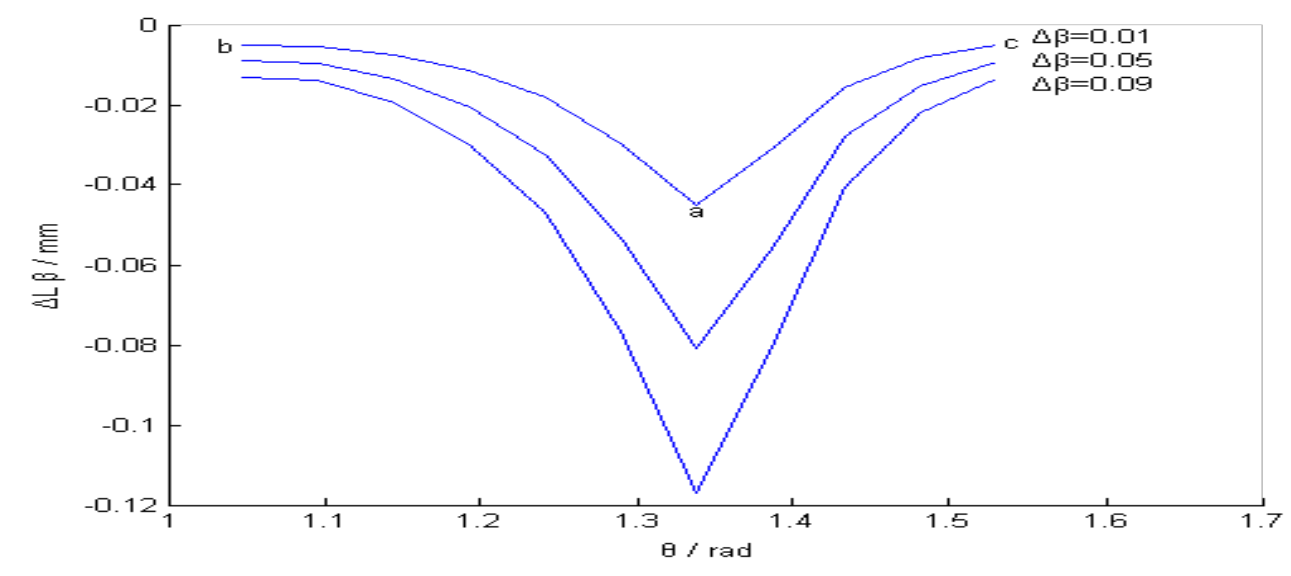

Fig.6 $\Delta L_{\beta}$ change curve

In summary, Fig. $3 \sim 6$ shows the influence degree of outer cycloid groove's structural parameter deviation on tooth profile error, and influence law of that to the shape changing of actual tooth profile. Fig. 3 and 5 show that the curves of influence of $\Delta e$ and $\Delta r$ on tooth profile error are similar in their shapes. Fig. 4 and 6 show that the curves of influence of $\Delta K$ and $\Delta \beta$ on tooth profile error are similar in their shapes. When the parameter errors are the same in values, the amplitude of variation $\Delta \mathrm{L}$ caused by $\Delta e$ and $\Delta \beta$ will be larger, and the whole tooth profile will have larger error, then the amplitude of variation $\Delta \mathrm{L}$ caused by $\Delta K$ will be larger than that caused by $\Delta r$. Therefore, the structural parameters e, $\beta$ and $\mathrm{k}$ are the most sensitive ones, their deviations must be strictly controlled in the design of cycloid steel ball planetary transmission, while the parameter $r$ is allowed to be free from this kind of strictly control. For inner cycloid groove, similar error changing law can be obtained through calculation and drawing.

\section{Conclusion}

(1) Equations (6) (9) are applicable to error analysis of cycloid steel ball planetary transmission.

(2) It can be learned from Fig. 3 6 that the tooth profile error can be greatly affected by $\Delta e, \Delta K$, $\Delta \beta, \Delta r$.

(3) Eccentricity e, steel ball radius $r$, curtate coefficient $k$, grooved angle $\beta$ are important parameters affecting tooth profile error; and deviations of e, $\beta$, k should be particularly controlled as 
possible in the design and processing of cycloid plate.

\section{References}

[1] QuJifang. (1993),Movable tooth transmission theory, China Machine Press.

[2] An Zijun, etc. (1996), Comprehensive study of tooth profile of cycloid steel ball transmission, Chinese Journal of Mechanical Engineering.

[3] QuJifang,AnZijun. (1994),Research on zero clearance cycloid ball transmission. Chinese Journal of Mechanical Engineering(English Edition), 7(1):17 22

[4] Zhang Shuying, Liang Guoming. (1986),Comprehensive analysis of processing error of cycloid pinwheel meshing gears, Journal of Mechanical Engineering

[5] Mao Yingtai. (1982),Deviation theory and accuracy Analysis,Defense Industry Press. 